\title{
Jumping to our defence
}

\author{
The first clinical success of immunotherapeutics for cancer treatment and the appreciation that \\ tissue regeneration can be greatly improved by precisely and locally modulating the immune \\ response are evidence that immunotherapy is poised to revolutionize the way we treat disease.
}

The immune system is not only our defence mechanism against pathogens, but is also crucial for tissue repair and regeneration. A dysfunctioning immune response can cause autoimmune diseases and allergies, and plays an important role in cancer surveillance and pathogenesis. An orchestra of cellular phenotypes and signalling pathways dynamically interact to guarantee a functioning immune system throughout life, offering a variety of intervention strategies to tackle immune failure or to modulate immune cell function. Immunotherapy aims to tailor and use immune cells and signalling molecules as therapeutics and has become a promising tool for the treatment of disease, as highlighted by the 2018 Nobel Prize for Physiology or Medicine awarded to James P. Allison and Tasuku Honjo for their discovery of cancer therapy by inhibition of negative immune regulation.

However, we are still far from understanding the details of the complex interplay between the different immune players or the mechanisms underlying immune tolerance and its relation to pathogenesis. This is partly owing to the lack of adequate models. Furthermore, systemic and adverse side-effects, caused by non-specific non-targeted drug delivery, as well as often low patient response rates remain major challenges in immunotherapy. This has sparked the interest of materials scientists and bioengineers to apply knowledge gained from decades of experience with nanomedicine, biomaterials and tissue engineering to design physiologically relevant in vitro and ex vivo models that allow the detailed investigation of the immune system and to develop platforms for the targeted delivery of immunotherapeutics and the precise manipulation of immune system components.

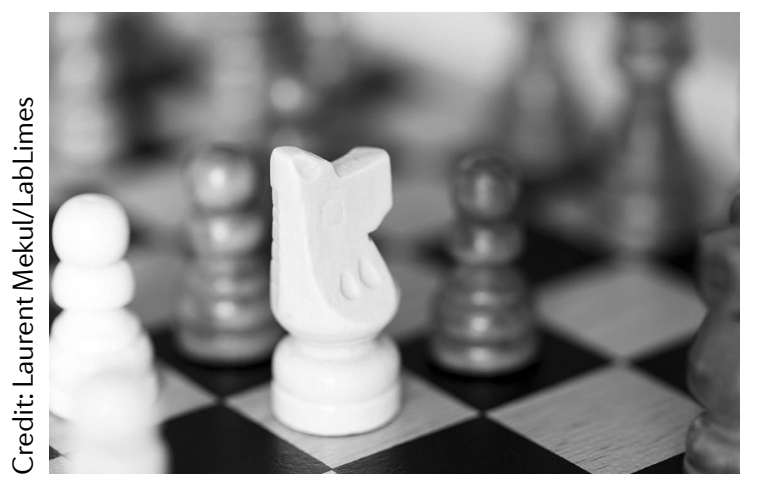

In our focus issue on immunoengineering, we explore a wide range of materials science strategies and challenges for immunotherapy. In a Comment, Elyse Watkins and Jeffrey Hubbell discuss how therapeutics can be endowed with designed biofunctionality, particularly in vaccinology, cancer immunotherapy and immune tolerance. In a Review, Susan Thomas and colleagues investigate how materials can be specifically engineered for lymph node drug delivery to target lymph noderesident cells. Multiscale engineering of immune cells and organs is examined by Ankur Singh and colleagues, highlighting material strategies to recreate primary, secondary and tertiary immune organs in vivo and in vitro. Cherie Stabler, Benjamin Keselowsky and colleagues discuss the design of immunomodulatory biomaterials for type 1 diabetes, with implications for other autoimmune diseases. David Mooney and colleagues consider macroscale biomaterials strategies to precisely control the delivery of pharmacological and cellular agents for tissue repair and cancer immunotherapy. How nanomedicine can be applied for combination cancer immunotherapy is discussed by James Moon and colleagues. Finally, in a Perspective, Yamuna Krishnan and colleagues explore the potential of synthetic DNA and RNA architectures to probe and programme immune cells.

The field of immunoengineering is progressing at high speed, and materials science greatly contributes to this progress by providing smart and controllable tools for immunotherapy with the aim to ultimately improve clinical outcomes. The success of preclinical nanomedicinebased combination cancer immunotherapies, the key role of biomaterials for islet transplantation and for the expansion and differentiation of immune cells ex vivo, and the possibility to use nanomaterials to deliver drugs to specific immune cell types, already demonstrate the importance of immunoengineering for immunotherapy. However, much remains to be fine-tuned and understood in immunoengineering, and close collaboration and communication of material scientists with immunologists and immuno-oncologists will be required to tailor engineering approaches to clinical needs. The research and strategies discussed in this focus issue illustrate not only the enthusiasm of materials scientists and engineers to contribute to immunotherapy, but also the immense range of possibilities they have to offer to tackle key questions in immunology and immunotherapy. 